\title{
A logarithmic estimate for harmonic sums and the digamma function, with an application to the Dirichlet divisor problem
}

\section{G.J.O. Jameson ${ }^{1 *}$ (D)}

\section{"Correspondence:}

g.jameson@lancaster.ac.uk

'Department of Mathematics and Statistics, Lancaster University, Lancaster, UK

\section{Springer}

\begin{abstract}
Let $H_{n}=\sum_{r=1}^{n} 1 / r$ and $H_{n}(x)=\sum_{r=1}^{n} 1 /(r+x)$. Let $\psi(x)$ denote the digamma function. It is shown that $H_{n}(x)+\psi(x+1)$ is approximated by $\frac{1}{2} \log f(n+x)$, where $f(x)=x^{2}+x+\frac{1}{3}$, with error term of order $(n+x)^{-5}$. The cases $x=0$ and $n=0$ equate to estimates for $H_{n}-\gamma$ and $\psi(x+1)$ itself. The result is applied to determine exact bounds for a remainder term occurring in the Dirichlet divisor problem.
\end{abstract}

MSC: Primary 26D15; 33B15; secondary 11N37

Keywords: Harmonic sum; Euler's constant; Digamma function; Divisor problem

\section{Introduction and summary of results}

Write $H_{n}$ for the harmonic sum $\sum_{r=1}^{n} \frac{1}{r}$. The following well-known estimation can be established by an Euler-Maclaurin summation, or by the logarithmic and binomial series:

$$
H_{n}-\gamma=\log n+\frac{1}{2 n}-\frac{1}{12 n^{2}}+r_{n}
$$

where $\gamma$ is Euler's constant and

$$
0<r_{n} \leq \frac{1}{120 n^{4}} .
$$

Since $\log \left(n+\frac{1}{2}\right)=\log n+\frac{1}{2 n}+O\left(\frac{1}{n^{2}}\right)$, it is a natural idea to absorb the term $\frac{1}{2 n}$ into the logarithmic term and compare $H_{n}-\gamma$ directly with $\log \left(n+\frac{1}{2}\right)$. This was done by De Temple [6]: he showed that

$$
H_{n}-\gamma=\log \left(n+\frac{1}{2}\right)+\frac{1}{24\left(n+\frac{1}{2}\right)^{2}}-r_{n}
$$

where

$$
\frac{7}{960(n+1)^{4}} \leq r_{n} \leq \frac{7}{960 n^{4}} .
$$

(We will repeatedly re-use the notation $r_{n}$ for the remainder term in estimations like this, with a new meaning each time.)

(c) The Author(s) 2019. This article is distributed under the terms of the Creative Commons Attribution 4.0 International License (http://creativecommons.org/licenses/by/4.0/), which permits unrestricted use, distribution, and reproduction in any medium, provided you give appropriate credit to the original author(s) and the source, provide a link to the Creative Commons license, and indicate if changes were made. 
Negoi [9] demonstrated that the $n^{-2}$ term can be absorbed into the log term by considering $\log h(n)$, where $h(n)=n+\frac{1}{2}+\frac{1}{24 n}$. His result is

$$
H_{n}-\gamma=\log h(n)-r_{n}
$$

where $\frac{1}{48}(n+1)^{-3} \leq r_{n} \leq \frac{1}{48} n^{-3}$. Numerous more recent articles have developed this process further. For example, Chen and Mortici [3] show that

$$
H_{n}-\gamma=\log \left(n+\frac{1}{2}+\frac{1}{24 n}-\frac{1}{48 n^{2}}+\frac{23}{5760 n^{3}}\right)+O\left(n^{-5}\right) .
$$

Further variations and extensions are given, for example, in [7] and [4], and in other references listed in these papers. One natural extension is the replacement of $H_{n}-\gamma$ by $\psi(x+1)$, where $\psi(x)$ is the digamma function $\Gamma^{\prime}(x) / \Gamma(x)$, since $H_{n}-\gamma=\psi(n+1)$.

A slightly different approach, which has proved quite effective, is to compare $H_{n}-\gamma$ or $\psi(x+1)$ with expressions of the form $\frac{1}{2} \log f(n)$. Using no more than the elementary inequalities $H_{n}-\gamma \leq \log n+\frac{1}{2 n}$ and $e^{x} \leq 1+x+x^{2}$, it is easily shown that $H_{n}-\gamma \leq \frac{1}{2} \log \left(n^{2}+\right.$ $n+1)$. With $h(n)$ as in (3), we have $h(n)^{2}=n^{2}+n+\frac{1}{3}+O\left(\frac{1}{n}\right)$, suggesting that the right comparison is with $f(n)=n^{2}+n+\frac{1}{3}$. Indeed, with $f(n)$ defined in this way, Batir [2] and $\mathrm{Lu}[5]$ have shown, by different methods, that

$$
H_{n}-\gamma=\frac{1}{2} \log f(n)-r_{n}
$$

where $r_{n} \sim 1 /\left(180 n^{4}\right)$. Lu obtained (5) as one case of a rather complicated analysis extending to further terms and parameters.

Our objective here is to refine and extend (5) by considering the sum

$$
H_{n}(x)=\sum_{r=1}^{n} \frac{1}{r+x}
$$

To identify the limit of $H_{n}(x)-\log n$, recall Euler's limit formula for the Gamma function: this can be written as $\Gamma(x+1)=\lim _{n \rightarrow \infty} G_{n}(x+1)$, where

$$
G_{n}(x+1)=\frac{n^{x}(n+1) !}{(x+1) \ldots(x+n)}
$$

from which it follows that $\lim _{n \rightarrow \infty}\left[H_{n}(x)-\log n\right]=-\psi(x+1)$. (No further facts about $\psi(x)$ are needed for our purposes.) We compare the difference $H_{n}(x)+\psi(x+1)$ with $\frac{1}{2} \log f(n+$ $x$ ). The case $x=0$ reproduces (5), while the case $n=0$ (with $H_{0}(x)=0$ ) gives a similar estimate for $\psi(x+1)$. Unlike [2] and [5], we give explicit upper and lower bounds for the error term, which we will require in the subsequent application. The exact statement is as follows.

Theorem 1 Let $n \geq 1$ and $x>-1$, or $n=0$ and $x>0$, and let $H_{n}(x)$ be defined by (6). Let $f(x)=x^{2}+x+\frac{1}{3}$. Then

$$
H_{n}(x)+\psi(x+1)=\frac{1}{2} \log f(n+x)-r(n, x),
$$


where

$$
\frac{1}{180(n+x+1)^{4}} \leq r(n, x) \leq \frac{1}{180(n+x)^{4}} .
$$

In particular,

$$
H_{n}-\gamma=\frac{1}{2} \log f(n)-r_{n},
$$

where

$$
\frac{1}{180(n+1)^{4}} \leq r_{n} \leq \frac{1}{180 n^{4}} .
$$

Also, for $x>0$,

$$
\psi(x+1)=\frac{1}{2} \log f(x)-r(x),
$$

where

$$
\frac{1}{180(x+1)^{4}} \leq r(x) \leq \frac{1}{180 x^{4}} .
$$

Our proof, given in Sect. 2, is a development of De Temple's original method.

Note that the difference between the upper and lower bounds in (10) is less than $1 / 45 n^{5}$. Of course, (9) is actually a special case of (11).

The case $x=-\frac{1}{2}$ in (7) leads to the following estimation of sums of odd reciprocals (which could not be derived from (9)). The proof is very short, so we include it here.

Corollary 1 Let $U_{n}=\sum_{r=1}^{n} \frac{1}{2 r-1}$. Then

$$
U_{n}-\frac{1}{2} \gamma-\log 2=\frac{1}{4} \log \left(n^{2}+\frac{1}{12}\right)-r_{n}
$$

where

$$
\frac{1}{360\left(n+\frac{1}{2}\right)^{4}} \leq r_{n} \leq \frac{1}{360\left(n-\frac{1}{2}\right)^{4}} .
$$

Proof Note that $2 U_{n}=H_{n}\left(-\frac{1}{2}\right)$. Also, $2 U_{n}=2 H_{2 n}-H_{n}$, from which it follows easily that $-\psi\left(\frac{1}{2}\right)=\lim _{n \rightarrow \infty}\left[2 U_{n}-\log n\right]=\gamma+2 \log 2$. Finally, $f\left(n-\frac{1}{2}\right)=n^{2}+\frac{1}{12}$.

Theorem 1 has a rather surprising application to an expression that arises in the Dirichlet divisor problem. Denote the divisor function by $\tau(n)$ and its summation function $\sum_{n \leq x} \tau(n)$ by $T(x)$. Write

$$
F(x)=x \log x+(2 \gamma-1) x
$$

and

$$
T(x)=F(x)+\Delta(x) .
$$


The most basic form of Dirichlet's theorem (e.g. [1, p. 59]) states that $\Delta(x)=O\left(x^{1 / 2}\right)$. The problem of determining the true order of magnitude of $\Delta(x)$ is the "Dirichlet divisor problem". Denote by $\theta_{0}$ the infimum of numbers $\theta$ such that $\Delta(x)=O\left(x^{\theta}\right)$. It was already shown by Voronoi in 1903 that $\theta_{0} \leq \frac{1}{3}$ (e.g. see [10, Sect. 1.6.4]). The estimate has been gradually reduced in a long series of studies: the current best value [8] is $\theta_{0} \leq \frac{131}{416}$.

Write $[x]$ for the integer part of $x$ and let

$$
B(x)=x-[x]-\frac{1}{2}
$$

the 1-periodic extension of the function $x-\frac{1}{2}$ on $[0,1)$. Further, let

$$
S(x)=2 \sum_{j \leq x^{1 / 2}} B\left(\frac{x}{j}\right) .
$$

Note that $|B(x)| \leq \frac{1}{2}$, and hence $|S(x)| \leq\left[x^{1 / 2}\right]$, for all $x>0$. A key, if small, step in the proof of Voronoi's theorem and later refinements is the statement

$$
\Delta(x)=-S(x)+q(x)
$$

where $q(x)$ is bounded. A version of the usual proof can be found in [10, Sect. 1.6.4]; if scrutinised carefully, it gives the bound 3 for $|q(x)|$. This is quite good enough for the purpose of proving Voronoi's theorem: the serious work is the estimation of $S(x)$ by exponential sums. However, it is still of some interest to determine the true bounds for $q(x)$, along with some other facts about its nature. We will see that $q(x)$ is continuous at integers, and that (9), together with (1), is exactly what is needed to establish:

Theorem 2 With $q(x)$ defined as above, we have, for all $x \geq 1$,

$$
-\frac{1}{6}<q(x)<\frac{1}{3}
$$

Both bounds are optimal.

\section{The proof of Theorem 1}

The key step is the following lemma.

Lemma 1 Let $f(x)=x^{2}+x+\frac{1}{3}$. Then, for all $x \geq 1$,

$$
\log f(x)-\log f(x-1)=\frac{2}{x}-\delta(x)
$$

where

$$
\frac{2}{45 x^{5}}<\delta(x)<\frac{2}{45\left(x-\frac{1}{2}\right)^{5}} .
$$

Proof We start with $f(x)=x^{2}+x+c$ and allow the choice of $c$ to emerge from the reasoning. Let $\delta(x)$ be defined by (15). Now $f(x) / f(x-1) \rightarrow 1$, and hence $\delta(x) \rightarrow 0$, as $x \rightarrow \infty$. So 
$\delta(x)=-\int_{x}^{\infty} \delta^{\prime}(t) d t$ for all $x>0$. Now

$$
\begin{aligned}
-\delta^{\prime}(t) & =\frac{2 t+1}{f(t)}-\frac{2 t-1}{f(t-1)}+\frac{2}{t^{2}} \\
& =\frac{G(t)}{t^{2} f(t) f(t-1)}
\end{aligned}
$$

where

$$
\begin{aligned}
G(t) & =t^{2}(2 t+1)\left(t^{2}-t+c\right)-t^{2}(2 t-1)\left(t^{2}+t+c\right)+2\left(t^{2}+t+c\right)\left(t^{2}-t+c\right) \\
& =-t^{2}\left(2 t^{2}-2 c\right)+2\left[t^{4}+(2 c-1) t^{2}+c^{2}\right] \\
& =2(3 c-1) t^{2}+2 c^{2} .
\end{aligned}
$$

To eliminate the $t^{2}$ term, we now choose $c=\frac{1}{3}$, so that $G(t)=\frac{2}{9}$ and

$$
-\delta^{\prime}(t)=\frac{2}{9 t^{2} f(t) f(t-1)} .
$$

Now $f(t) f(t-1)=t^{4}-\frac{1}{3} t^{2}+\frac{1}{9}<t^{4}$ for $t \geq 1$, so

$$
\delta(x)>\int_{x}^{\infty} \frac{2}{9 t^{6}} d t=\frac{2}{45 x^{5}}
$$

for $x \geq 1$. On the other hand, $f(t)>f(t-1)>\left(t-\frac{1}{2}\right)^{2}$, hence

$$
\delta(x)<\int_{x}^{\infty} \frac{2}{9\left(t-\frac{1}{2}\right)^{6}} d t=\frac{2}{45\left(x-\frac{1}{2}\right)^{5}} .
$$

Proof of Theorem 1 Apply the identity (15) to $r+x$ for $1 \leq r \leq n$ and add: we find

$$
\log f(n+x)-\log f(x)=2 H_{n}(x)-\sum_{r=1}^{n} \delta(r+x)
$$

equivalently

$$
2 H_{n}(x)-\log f(n+x)=-\log f(x)+\sum_{r=1}^{n} \delta(r+x) .
$$

Now $f(n+x) / n^{2} \rightarrow 1$, so $\log f(n+x)-2 \log n \rightarrow 0$, as $n \rightarrow \infty$. Taking the limit in (17), we see that

$$
-2 \psi(x+1)=-\log f(x)+\sum_{r=1}^{\infty} \delta(r+x) .
$$

Now taking the difference, we have

$$
2 H_{n}(x)-\log f(n+x)+2 \psi(x+1)=-2 r(n, x),
$$


where

$$
2 r(n, x)=\sum_{r=n+1}^{\infty} \delta(r+x) .
$$

The condition $x>-1$ ensures that the inequality (16) applies to $\delta(r+x)$ for $r \geq 2$. By integral estimation, we now have, for $n \geq 1$,

$$
r(n, x) \geq \sum_{r=n+1}^{\infty} \frac{1}{45(r+x)^{5}}>\int_{n+1}^{\infty} \frac{1}{45(t+x)^{5}} d t=\frac{1}{180(n+x+1)^{4}} .
$$

At the same time,

$$
r(n, x) \leq \sum_{r=n+1}^{\infty} \frac{1}{45\left(r-\frac{1}{2}+x\right)^{5}} .
$$

The function $1 /(t+x)^{5}$ is convex, and convex functions $h(t)$ satisfy $h\left(y-\frac{1}{2}\right) \leq \int_{y-1}^{y} h(t) d t$, hence

$$
r(n, x) \leq \int_{n}^{\infty} \frac{1}{45(t+x)^{5}} d t=\frac{1}{180(n+x)^{4}} .
$$

For $x>0$, the case $n=0$ follows similarly from (18) (note that (16) now applies also to $\delta(1+x))$.

Note 1 The upper bounds for $\delta(x)$ in Lemma 1 and $r(n, x)$ in Theorem 1 can be slightly improved. One can verify that $t^{2} f(t) f(t-1) \geq\left(t-\frac{1}{12}\right)^{6}$ where we previously used $\left(t-\frac{1}{2}\right)^{6}$. This leads to $\delta(x)<2 /\left[45\left(x-\frac{1}{12}\right)^{5}\right]$ and $r(n, x) \leq 1 /\left[180\left(n+x+\frac{5}{12}\right)^{4}\right]$.

Note 2 In principle, one could derive Stirling-type approximations to $\log \Gamma(x)$ and $\Gamma(x)$ from (11), but only in terms of the rather unpleasant antiderivative of $\log f(x)$.

\section{The remainder in the divisor problem}

We return to the divisor problem. The starting point is Dirichlet's hyperbola identity [1, p. 59]:

$$
T(x)=2 \sum_{j \leq x^{1 / 2}}\left[\frac{x}{j}\right]-\left[x^{1 / 2}\right]^{2} .
$$

With our previous notation, note that

$$
T(x)+S(x)=F(x)+q(x) .
$$

Write $I_{n}=\left[n^{2},(n+1)^{2}\right]$.

Lemma 2 For $x \in I_{n}$,

$$
q(x)=x H_{n}-F(x)-n(n+1) .
$$

The function $q(x)$ is continuous for all $x \geq 1$, and concave on each interval $I_{n}$. 
Proof We work with $T(x)+S(x)$. For $n^{2} \leq x<(n+1)^{2}$, we have by (19)

$$
\begin{aligned}
T(x) & =2 \sum_{j=1}^{n}\left(\frac{x}{j}-B\left(\frac{x}{j}\right)-\frac{1}{2}\right)-n^{2} \\
& =2 x H_{n}-S(x)-n-n^{2},
\end{aligned}
$$

which equates to (20). We check that this remains valid at $x=(n+1)^{2}$. By what we have just shown, with $n$ replaced by $n+1$,

$$
\begin{aligned}
T\left[(n+1)^{2}\right]+S\left[(n+1)^{2}\right] & =2 H_{n+1}(n+1)^{2}-(n+1)(n+2) \\
& =2 H_{n}(n+1)^{2}+2(n+1)-(n+1)(n+2) \\
& =2 H_{n}(n+1)^{2}-n(n+1),
\end{aligned}
$$

agreeing with (20). Hence $F(x)+q(x)$, and consequently $q(x)$ itself, is continuous for all $x \geq 1$. Also, $q^{\prime}(x)=2 H_{n}-F^{\prime}(x)=2 H_{n}-\log x-2 \gamma$, which is decreasing, so $q(x)$ is concave on $I_{n}$.

So in fact $F(x)+q(x)$ is linear on $I_{n}$. For example,

$$
F(x)+q(x)= \begin{cases}2 x-2 & \text { for } 1 \leq x \leq 4 \\ 3 x-6 & \text { for } 4 \leq x \leq 9\end{cases}
$$

The reason for continuity of $T(x)+S(x)$ is easily seen directly. At non-square integers $k$, $T(x)$ increases by $\tau(k)$. Meanwhile, for each divisor $j$ of $k$ with $j<k^{1 / 2},[x / j]$ increases by 1 , so $B(x / j)$ decreases by 1 . There are $\frac{1}{2} \tau(k)$ such divisors $j$, so $S(x)$ decreases by $\tau(k)$. At square integers $k=n^{2}$, the new term $2 B(k / n)=-1$ enters the sum, so again the decrease in $S(x)$ is $\tau(k)$.

To determine the lower bound of $q(x)$, we consider $q\left(n^{2}\right)$ and apply (1).

Lemma 3 We have $q(x)>-\frac{1}{6}$ for all $x \geq 1$. Further, $q\left(n^{2}\right) \leq-\frac{1}{6}+\frac{1}{60 n^{2}}$, so $\inf _{x \geq 1} q(x)=-\frac{1}{6}$.

Proof By (20) and (1) (with $r_{n}$ as in (1)),

$$
\begin{aligned}
q\left(n^{2}\right) & =2 n^{2} H_{n}-2 n^{2} \log n-(2 \gamma-1) n^{2}-n-n^{2} \\
& =2 n^{2}\left(H_{n}-\log n-\gamma\right)-n \\
& =2 n^{2}\left(\frac{1}{2 n}-\frac{1}{12 n^{2}}+r_{n}\right)-n \\
& =-\frac{1}{6}+2 n^{2} r_{n} .
\end{aligned}
$$

So

$$
-\frac{1}{6}<q\left(n^{2}\right) \leq-\frac{1}{6}+\frac{1}{60 n^{2}} .
$$


This applies also at $(n+1)^{2}$. Since $q(x)$ is concave on $I_{n}$, it follows that $q(x)>-\frac{1}{6}$ throughout this interval.

Finally, we apply (9) and (10) to identify the upper bound.

Proof of the upper bound in Theorem 2 Let $x_{n}$ be the point where $q(x)$ attains its maximum in $I_{n}$. Since $q^{\prime}(x)=2 H_{n}-\log x-2 \gamma$, we have $\log x_{n}=2 H_{n}-2 \gamma$, hence the maximum value is

$$
\begin{aligned}
q\left(x_{n}\right) & =2 H_{n} x_{n}-F\left(x_{n}\right)-n(n+1) \\
& =\left(\log x_{n}+2 \gamma\right) x_{n}-F\left(x_{n}\right)-n(n+1) \\
& =x_{n}-n(n+1) .
\end{aligned}
$$

By (9) and (10) (using only $r_{n}>0$ ), we have $\log x_{n}<\log f(n)$, so $x_{n}<f(n)=n(n+1)+\frac{1}{3}$, hence $q\left(x_{n}\right)<\frac{1}{3}$.

We show that, conversely,

$$
q\left(x_{n}\right)>\frac{1}{3}-\frac{1}{45 n^{2}}
$$

for $n \geq 2$, so that $\sup _{x \geq 1} q(x)=\frac{1}{3}$. By (21), this is equivalent to $x_{n}>f(n)-\frac{1}{45 n^{2}}$. By $(10)$,

$$
\log x_{n}=2 H_{n}-2 \gamma \geq \log f(n)-\frac{1}{90 n^{4}}
$$

Now using the inequality $e^{-x} \geq 1-x$, together with $f(n) \leq 2 n^{2}$, we have

$$
x_{n} \geq f(n) e^{-1 / 90 n^{4}} \geq f(n)\left(1-\frac{1}{90 n^{4}}\right) \geq f(n)-\frac{1}{45 n^{2}} .
$$

Acknowledgements

Not applicable.

Funding

No funding received.

Availability of data and materials

Not applicable.

\section{Competing interests}

The author declares that he has no competing interests.

Author's contributions

The author read and approved the final manuscript.

\section{Publisher's Note}

Springer Nature remains neutral with regard to jurisdictional claims in published maps and institutional affiliations.

Received: 11 March 2019 Accepted: 17 May 2019 Published online: 27 May 2019

\section{References}

1. Apostol, T.M.: Introduction to Analytic Number Theory. Springer, Berlin (1976)

2. Batir, N.: Sharp bounds for the psi function and harmonic numbers. Math. Inequal. Appl. 14, 917-925 (2011)

3. Chen, C., Mortici, C.: New sequence converging towards the Euler-Mascheroni constant. Comput. Math. Appl. 64 391-398 (2012) 
4. Dawei, L.: Some new convergent sequences and inequalities of Euler's constant. J. Math. Anal. Appl. 419, 541-552 (2014)

5. Dawei, L.: Some quicker classes of sequences convergent to Euler's constant. Appl. Math. Comput. 232, 172-177 (2014)

6. De Temple, D.W.: A quicker convergence to Euler's constant. Am. Math. Mon. 100, 468-470 (1993)

7. Elezovic, N.: Estimations of psi function and harmonic numbers. Appl. Math. Comput. 258, 192-205 (2015)

8. Huxley, M.N.: Exponential sums and lattice points III. Proc. Lond. Math. Soc. (3) 87, 591-609 (2003)

9. Negoi, T.: A faster convergence to Euler's constant. Math. Gaz. 83, 487-489 (1999)

10. Tenenbaum, G.: Introduction to Analytic and Probabilistic Number Theory. Cambridge Univ. Press, Cambridge (1995)

Submit your manuscript to a SpringerOpen ${ }^{\odot}$ journal and benefit from:

- Convenient online submission

- Rigorous peer review

- Open access: articles freely available online

- High visibility within the field

- Retaining the copyright to your article

Submit your next manuscript at $\gg$ springeropen.com 